\title{
Simulation of Convection and Macrosegregation in a Large Steel Ingot
}

\author{
J.P. GU and C. BECKERMANN
}

\begin{abstract}
Melt convection and macrosegregation in casting of a large steel ingot are numerically simulated. The simulation is based on a previously developed model for multicomponent steel solidification with melt convection and involves the solution of fully coupled conservation equations for the transport phenomena in the liquid, mush, and solid. Heat transfer in the mold and insulation materials, as well as the formation of a shrinkage cavity at the top, is taken into account. The numerical results show the evolution of the temperature, melt velocity, and species concentration fields during solidification. The predicted variation of the macrosegregation of carbon and sulfur along the vertical centerline is compared with measurements from an industrial steel ingot that was sectioned and analyzed. Although generally good agreement is obtained, the neglect of sedimentation of free equiaxed grains prevents the prediction of the zone of negative macrosegregation observed in the lower part of the ingot. It is also shown that the inclusion of the shrinkage cavity at the top and the variation of the final solidification temperature due to macrosegregation is important in obtaining good agreement between the predictions and measurements.
\end{abstract}

\section{INTRODUCTION}

MACROSEGREGATION in large steel ingots is one of the most well-known and now classical problems in the field of solidification and casting. ${ }^{[1]}$ It arises from the relative movement of solute-rich or poor liquid and solid phases during solidification over distances much larger than the dendrite arm spacings. Commonly found macrosegregation patterns in steel ingots are a positively segregated zone at the top, negative segregation near the bottom in the equiaxed zone, inverse segregation near the ingot surface, $\mathrm{V}$ segregates along the centerline, and A segregates in the columnar zone. $^{[2,3]}$ These inhomogeneities can severely limit the yield in ingot casting and cause problems in the subsequent processing and final steel properties. It is well established that the macroscopic transport of species leading to macrosegregation can occur by a variety of mechanisms, including thermally and solutally driven natural convection of the melt in the mushy zone, flow due to solidification contraction, and the sedimentation of free equiaxed crystals. Fredriksson and co-workers ${ }^{[4,5]}$ provide recent overviews of the solidification, transport phenomena, and macrosegregation in ingot casting.

While there exists a relatively good understanding of the physical processes that cause macrosegregation in steel ingots, the mathematical modeling and quantitative numerical prediction of macrosegregation have proven to be difficult. Macrosegregation models were first developed by Flemings and co-workers. ${ }^{[6,7]}$ These models have yielded much insight into macrosegregation due to interdendritic fluid flow. Ohnaka ${ }^{[8]}$ presented a numerical model for predicting macrosegregation in steel ingots, but it was limited to a binary Fe-C alloy. In the study by Olsson et al. ${ }^{[5]}$ a simple ingot macrosegregation model was developed that

J.P. GU, Postdoctoral Researcher, and C. BECKERMANN, Professor, are with the Department of Mechanical Engineering, University of Iowa, Iowa City, IA 52242.

Manuscript submitted July 31, 1998. included consideration of the settling of free crystals and A segregates. Although good agreement with experiments was achieved, the model relied on many approximations (such as estimating the flow velocities). It should also be mentioned that many models have appeared in the literature that are solely concerned with heat transfer during steel ingot casting (for example, Reference 9 and references therein). While these models form the foundation for any realistic ingot casting modeling, they do not address solid/liquid transport during solidification and the resulting macrosegregation.

More recently, macrosegregation models have been formulated that rely on fully coupled numerical solutions of the mass, momentum, energy, and species conservation equations for a solid/liquid mixture. ${ }^{[10,11]}$ Beckermann and Wang $^{[12]}$ and Prescott and Incropera ${ }^{[13]}$ have reviewed the progress in this area. Although many basic phenomena, such as the formation of A segregates and the negative segregation due to settling of equiaxed crystals, have been successfully simulated on laboratory-scale systems, the application of such continuum or multiphase models to multicomponent steel ingot solidification has been very limited.

Combeau and co-workers ${ }^{[14-17]}$ attempted to extend recent binary alloy solidification models that couple mass, momentum, energy, and species conservation in all regions (solid, mush, and bulk liquid) to model steel solidification considering only buoyancy driven flow. In order to simplify the calculation procedure, the mass, momentum, and energy conservation equations were solved assuming that the compositions in the mushy zone were given by the lever rule (for carbon) or Scheil equation (for other elements). With the solid fraction and velocity distribution known, the solid and liquid compositions were then determined explicitly at the end of each time-step. Using a binary Fe-C alloy solidifying in a cylindrical ingot, Vannier et al. ${ }^{[17]}$ compared this "partially coupled" method of calculation with fully coupled predictions. While the two methods predict the same trends, differences of up to 70 pct were apparent in axial segregation profiles, and at some locations along the centerline, the 
partially coupled model predicted positive segregation where the coupled model indicated negative segregation. Combeau et $a{ }^{[15]}{ }^{[1]}$ used the model to examine the effects of initial and boundary conditions on the formation of macrosegregation and found that the amount of superheat had little effect on the predicted carbon segregation along the ingot centerline, while insulating the top of the cylindrical ingot reduced the degree of carbon segregation at the ingot top. Roch et al. ${ }^{[14,16]}$ determined that the predicted degree of carbon segregation along the axis of a cylindrical steel ingot decreased as the specified mushy-zone permeability was decreased, decreased when the specified value of the carbon-partition coefficient was increased, and decreased as the height-todiameter ratio of the ingot was increased. In addition, slightly increasing the molybdenum content of the steel (from 0 to $1 \mathrm{wt}$ pct Mo) was shown to decrease the extent of carbon segregation along the ingot axis, but a drastic increase in the molybdenum content ( $3 \mathrm{wt}$ pct Mo) actually increased the carbon segregation. Finally, Vannier et al. ${ }^{[17]}$ obtained qualitative agreement between measurements and predictions of the evolution of carbon segregation and the final carbon distribution along the centerline of a 65-ton cylindrical steel ingot.

The objective of the present study is to apply a recently developed micro-/macrosegregation model for multicomponent steels ${ }^{[18]}$ to casting of a production-scale steel ingot and compare the predictions to measurements of the segregation pattern. The model involves the simultaneous numerical solution of the fully coupled mass, momentum, energy, and species (for each element) conservation equations in the liquid, solid, and mushy regions, and the heat conduction equation in the cast iron mold and hot top, together with realistic boundary conditions. The effects of each element in the steel on the liquidus temperature, partition coefficients and liquidus slopes, back-diffusion and solid fraction evolution, liquid density and buoyancy forces are fully accounted for. Furthermore, a procedure is implemented in the model that calculates the drop in the liquid steel level and the formation of the shrinkage cavity at the top due to thermal contraction of the liquid during cooling and the shrinkage associated with the liquid-to-solid transformation.

As in the original model, ${ }^{[18]}$ the movement of solid in the form of settling equiaxed crystals is neglected. Although models have been proposed that include the effects of nonstationary equiaxed crystals, ${ }^{[12]}$ they are of a very preliminary nature and cannot be used with sufficient confidence in the modeling of steel ingot solidification. In particular, the generation and growth of equiaxed crystals in an undercooled convecting melt cannot presently be modeled accurately. ${ }^{[12]}$ The large uncertainties introduced by such a preliminary model could, in fact, prevent a meaningful comparison with measurements. The neglect of settling equiaxed crystals can be expected to result in problems predicting the negative region of macrosegregation often observed near the ingot bottom. By comparing measurements and predictions, the present study will allow for an examination of the importance of the settling effect on the macrosegregation pattern. As demonstrated in Schneider and Beckermann, ${ }^{[18]}$ the model is, in principle, able to predict the formation of A segregates. However, the relative coarseness of the numerical grid prevents the prediction of these small-scale features in the present simulation. A segregates can have a strong effect on the overall macrosegregation pattern in the ingot, because liquid highly enriched in alloying elements streams through the A segregation channels in the mush out into the bulk liquid. ${ }^{[4]}$ Similar to the issue of the settling equiaxed crystals, the present comparison with the measurements may shed light on the importance of this effect. Hence, the simulation reported here should only be viewed as preliminary in some respects and in need of improvement, once improved models and greater computational resources become available. Nonetheless, it represents an accurate assessment of the current predictive capabilities of fully coupled solidification/segregation models, with essentially no adjustable parameters, when applied to a full-scale steel ingot.

\section{DESCRIPTION OF THE STEEL INGOT SIMULATED}

The ingot simulated in the present study was cast, sectioned, and analyzed by Lukens Steel Company (Coatesville, PA). It was a large 1.016-m-wide $\times 2.083$-m-deep $\times 2.819$ $\mathrm{m}$-high big end up ingot of AISI grade 4142 steel. The ladle composition of this ingot is given in Table I. The cast iron mold had a taper of 11 pct, such that the ingot was 1.016$\mathrm{m}$ wide at the top and $0.705-\mathrm{m}$ wide at the bottom. The thickness of the cast iron mold varied from 0.337 to 0.276 $\mathrm{m}$. The narrow faces of the mold were corrugated.

The ingot was cast with a 14 pct volume hot top consisting of 3.81-cm-thick low density insulating sideboards, bottom pour casting flux, and an exothermic topping compound. The layer of combination flux and hot topping compound on top of the ingot at the conclusion of teaming was about 12.7-cm thick.

Teaming temperature for this ingot was $1807 \mathrm{~K}$ with about a $14 \mathrm{~K}$ temperature drop expected from ladle to mold during teeming. The calculated liquidus temperature is $1762 \mathrm{~K}$ using the phase-diagram data published by Kagawa and Okamoto. ${ }^{[19]}$ The ingot was sectioned along the centerline of the ingot broad face (i.e., a vertical cut at middepth) and machined. Fluorescent dye penetrant testing and sulfur prints revealed the presence of numerous inverted A segregates and an equiaxed center core. Drillings were obtained in a grid pattern along the vertical centerline and across the hot top of the cut section for the macrosegregation measurements. The drillings were analyzed for carbon and sulfur using a LECO* CS analyzer. Positive macrosegregation for

*LECO is a trademark of LECO Corporation, St. Joseph, MI.

carbon and sulfur was found to be 34 and 46 pct, respectively, at the hot top junction. The percentages refer to the relative difference to the ladle concentration of an element. The carbon concentration decreased to the ladle concentration at about $0.838 \mathrm{~m}$ below the junction, and the negative macrosegregation in the lower part was no more than 10 pct. More detailed measured macrosegregation patterns are presented subsequently.

\section{MODEL}

The model is restricted to the same vertical section at middepth as where the ingot was cut for the concentration measurements. Thus, the model is two-dimensional and 
Table I. Ladle Compostion of the Ingot (Weight Percent)

\begin{tabular}{cccccccccccc}
\hline $\mathrm{C}$ & $\mathrm{Mn}$ & $\mathrm{P}$ & $\mathrm{S}$ & $\mathrm{Cu}$ & $\mathrm{Si}$ & $\mathrm{Ni}$ & $\mathrm{Cr}$ & $\mathrm{Mo}$ & $\mathrm{V}$ & $\mathrm{Al}$ \\
\hline 0.41 & 0.79 & 0.012 & 0.035 & 0.13 & 0.24 & 0.18 & 0.90 & 0.17 & 0.05 & 0.01 \\
\hline
\end{tabular}

assumes an infinite ingot depth normal to the section. While this assumption is necessitated by the excessive computer costs associated with a three-dimensional analysis, it can be expected to result in some inaccuracies due to the neglect of heat losses and melt friction at the narrow faces of the ingot. Furthermore, the corrugations on the mold walls are neglected and symmetry is assumed at the centerline of the vertical section. The resulting simulation domain is shown in Figure 1, where all dimensions are in millimeters. Four different materials are present: the cast iron mold and base, the insulating boards, the flux and hot topping compound at the top, and the steel. The thermophysical properties of all materials except for steel are listed in Table II. They were obtained from Lukens Steel Company and can only be viewed as first approximations. The thermophysical properties of the steel and all phase-diagram parameters (partition coefficients, liquidus slopes, etc.) used in the simulation are essentially the same as those listed in Schneider and Beckermann $^{[18]}$ and are not repeated here. Again, many of the steel properties used are only accurate to within about

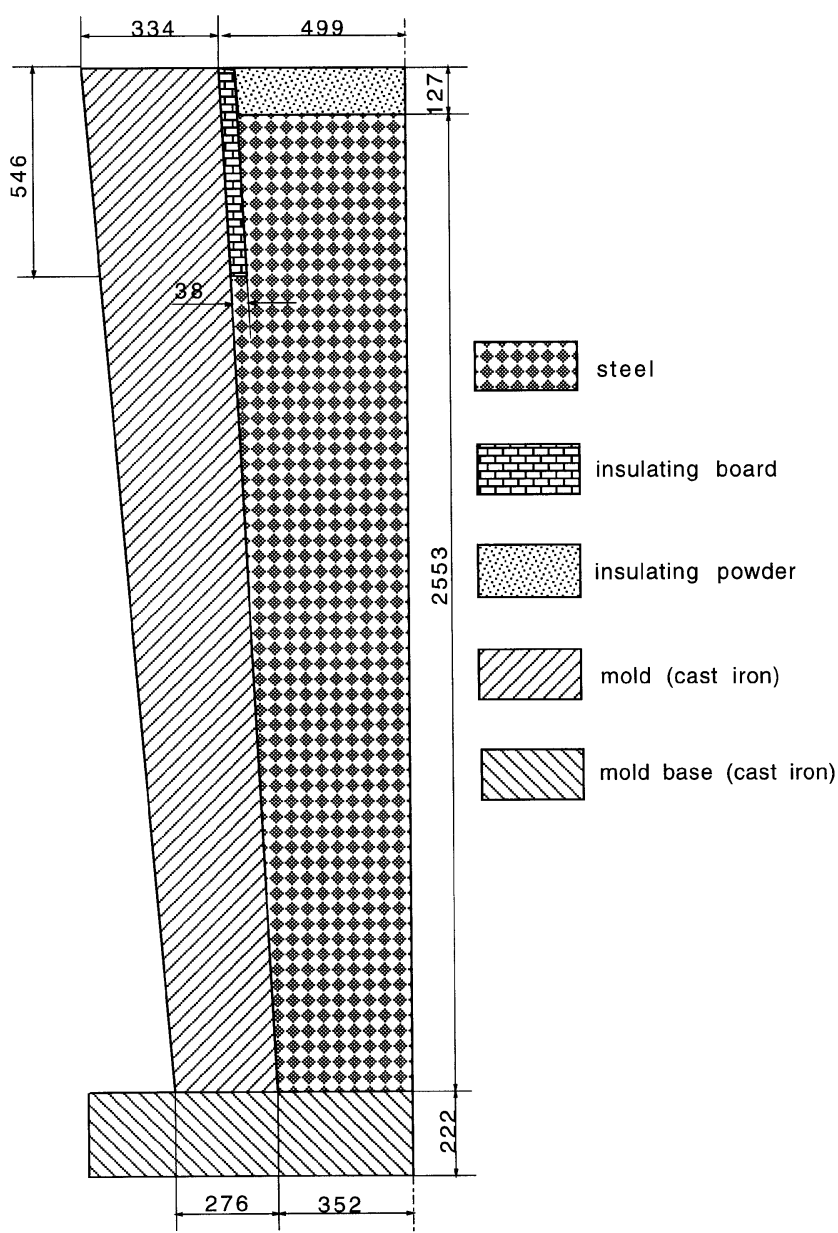

Fig. 1.-Schematic of the two-dimensional ingot cross section simulated showing all dimensions (in $\mathrm{mm}$ ) and materials.
20 pct, but it is felt that the resulting inaccuracies are well within the overall model uncertainty. Filling of the mold was not simulated, and the melt was assumed to be initially quiescent at a temperature of $1793 \mathrm{~K}$ (about $31 \mathrm{~K}$ superheat). All other materials are assumed to be initially at an ambient temperature of $303 \mathrm{~K}$. For the purpose of calculating the shrinkage cavity at the top, the liquid steel is assumed to contract by about 2 pct per $100 \mathrm{~K}$, and the solidification contraction is about 3 pct.

The governing conservation equations for mass, momentum, energy, and species are identical to those in Table I of Schneider and Beckermann, ${ }^{[18]}$ and only a brief description is provided here. The conservation equations are based on averaging the point equations within a phase over a representative elementary volume (REV) that contains arbitrary fractions of solid and liquid phases. They account for the microscopic flow and phase-change process in the mushy zone, while reducing to the correct single-phase limits in the pure solid and liquid regions. The solid is assumed to be rigid and stationary, the solid and liquid phases within a REV are assumed to be in thermal equilibrium, and the liquid within a REV is considered to be well mixed for all species. In the solid, as well as in the mold and insulating materials, the energy equation reduces to the regular transient heat conduction equation. Separate averaged species conservation equations are solved for each alloying element (11 elements total, Table I) in both the liquid and solid phases. The temperatures and liquid concentrations in the mushy zone, coupled with the phase-diagram relations, provide the local liquid fraction according to a method described in Reference 18.

The species equations incorporate a back-diffusion model to account for microsegregation, a detailed description of which can be found in Schneider and Beckermann. ${ }^{[18]}$ The back-diffusion model relies on a one-dimensional platelike secondary dendrite arm geometry, with the back-diffusion rate being proportional to the solid mass diffusivity of the element in question, the interfacial area per unit volume, and the inverse of a diffusion length that is based on a parabolic species concentration profile in a dendrite arm. With this model, the solidification path and final solidification temperature are calculated at each point within the ingot and will vary depending on the local cooling conditions and macrosegregation. All previous studies assume a certain solidification path and solidus temperature. As is illustrated subsequently, the so-called solidus temperature (defined here as the temperature at which the solid fraction reaches unity) can vary by more than $50 \mathrm{~K}$ in the ingot, because macrosegregation changes the local steel composition. The back-diffusion model requires the specification of the secondary dendrite arm spacing. This spacing is calculated from a relation given in Jacobi and Schwerdtfeger, ${ }^{[20]}$ and averages about $400 \mu \mathrm{m}$ for the present ingot simulation.

The liquid momentum equation reduces to the regular Navier-Stokes equation in the single-phase liquid region. In the mushy zone, the momentum equation contains the 
Table II. Thermophysical Properties of the Insulating Powder, Insulating Board, and Cast Iron

\begin{tabular}{|c|c|c|c|}
\hline Material & Density $\left(\mathrm{kg} / \mathrm{m}^{3}\right)$ & Specific Heat $(\mathrm{J} / \mathrm{kg} \cdot \mathrm{K})$ & $\begin{array}{l}\text { Thermal Conductivity } \\
(\mathrm{W} / \mathrm{m} \cdot \mathrm{K})\end{array}$ \\
\hline Insulating powder & 304.4 & 134.0 & $0.3201(0.2249$ after $45 \mathrm{~min})$ \\
\hline Insulating board & 480.6 & 134.0 & $0.9516(0.3288$ after $10 \mathrm{~min})$ \\
\hline Cast iron & 7300.0 & 850.0 & 37.0 \\
\hline
\end{tabular}

liquid volume fraction and a permeability term, that accounts for the friction the melt experiences as it flows through the dendritic network and forces the melt velocity to vanish as the liquid fraction approaches zero. The local permeability, $K$, is calculated from the following relation:

$$
K=6 \times 10^{-4} \lambda_{1}^{2} \frac{\varepsilon_{l}^{3}}{\left(1-\varepsilon_{l}\right)^{2}}
$$

where $\lambda_{1}$ is the primary dendrite arm spacing and $\varepsilon_{l}$ is the liquid volume fraction. This relation was found to give good agreement with previous experimental and analytical results (Figure 4 in Reference 21). The primary spacing is calculated from a relation given in Reference 20 and averages about $1200 \mu \mathrm{m}$ for the present ingot simulation. Finally, it should be mentioned that the momentum equation contains a buoyancy term, in which the liquid density is calculated as a function of the local temperature and concentration of each element in the liquid. These and other details can be found in Schneider and Beckermann ${ }^{[18]}$ and Schneider et al. ${ }^{[21]}$

A critical part in realistically simulating steel ingot casting is the specification of the thermal boundary conditions at the outer surfaces and the heat transfer coefficient due to gap formation at the mold-ingot interface. Many studies have been performed on this subject, and we follow an approach similar to that described in Thomas et al. ${ }^{[9]}$ The heat flux, $q$, at the vertical outer mold surface is given by the sum of radiation and natural convection contributions as

$$
q=\sigma \varepsilon_{m}\left(T_{s}^{4}-T_{\infty}^{4}\right)+1.24\left(T_{s}-T_{\infty}\right)^{1.33}
$$

where $\sigma=5.776 \times 10^{-8} \mathrm{~W} / \mathrm{m}^{2} \mathrm{~K}$; the emissivity of the mold surface, $\varepsilon_{m}$, is assumed to be 0.85 ; and $T_{s}$ and $T_{\infty}$ are the mold surface and ambient temperatures, respectively. At the top and bottom surfaces, for simplicity, Newton's law of cooling with constant heat transfer coefficients equal to 10 and $80 \mathrm{~W} / \mathrm{m}^{2} \mathrm{~K}$, respectively, was used. The centerline is adiabatic. For the purpose of estimating the gap heat flux, $q_{\text {gap }}$, the time for gap formation for the present ingot was estimated to be about 5400 seconds. ${ }^{[9]}$ After gap formation, the heat transfer across the gap is primarily by radiation and can be estimated from the following equation:

$$
q_{\mathrm{gap}}=\frac{\sigma\left(T_{s i}^{4}-T_{s m}^{4}\right)}{\frac{1}{\varepsilon_{i}}+\frac{1}{\varepsilon_{m}}-1}
$$

where $\varepsilon_{i}$ is the emissivity of the ingot surface (assumed equal to 0.9 ), and $T_{s i}$ and $T_{s m}$ are the surface temperatures of the ingot and mold, respectively, on either side of the gap. Before gap formation, the conductive heat transfer coefficient (or contact conductance) is assumed to drop linearly with time from an initial value of $3 \times 10^{8} \mathrm{~W} / \mathrm{m}^{2} \mathrm{~K}$ to zero at the time of gap formation. Again, the preceding thermal boundary conditions are empirically established and found to work well in other ingot casting simulations (Reference 9 and references therein).

The numerical solution procedures are described in Schneider and Beckermann. ${ }^{[18]}$ Essentially, the same set of governing equations is solved everywhere on a fixed, rectangular grid using a fully implicit method and the Simpler algorithm. The sloped material and domain boundaries are thus approximated as staircase patterns. Depending on the local fraction liquid and a material designator, the equations and thermophysical properties take on the correct values as described in the previous publication. ${ }^{[18]}$

The formation of a shrinkage cavity at the top of the ingot is considered in the present simulation. The total shrinkage volume (including liquid shrinkage and solidification contraction) is calculated after each time-step and summed. Once the shrinkage volume exceeds the volume of a grid cell that (1) contains steel, (2) has a solid fraction less than 0.05 , and (3) is closest to the top and centerline, that cell is considered empty and a new summation is started. The properties of an empty cell are taken to be those of the insulation compound. No other modifications were made to the governing equations published in Reference 18 .

A grid of $38 \times 54$ control volumes was used in the simulation reported here. About $27 \times 46$ control volumes are present in the ingot, while the remainder are distributed in the mold and insulating materials. At the beginning of solidification (before 7000 seconds), the time-step was about 0.15 seconds. As the convection inside the ingot becomes weaker, the time-step was increased up to 5 seconds. The total cpu time is difficult to estimate because the simulation was run on a variety of workstations, but is estimated at several weeks of equivalent cpu time on an HP* J200 single

*HP is a trademark of Hewlett-Packard Company, Colorado Springs, CO.

processor workstation. This long simulation time is primarily caused by the fact that the model equations are extremely complex (14 highly coupled advection-diffusion equations), the time-step is small relative to the total simulation time (of about 25,000 seconds), and the iterative solution algorithm is difficult to converge. Aside from the convergence issues discussed in Reference 18, some additional convergence problems seemed to arise from the presence of the sloped material boundaries. These problems were ultimately solved, and the cpu time per time-step was found to be similar to that reported in Reference 18 for a much smaller physical domain size. Due to the large size of the present ingot, the total time that was simulated was about 20 times greater than the one in Reference 18, while the time-step was about the same. Since the cpu time increases linearly with the number of time-steps, the aforementioned very long total cpu time resulted. Pure heat conduction simulations of the same ingot and with the same grid were found to require less than 4 hours of cpu time. Clearly, the bulk of the cpu 
time is due to the flow. It is difficult to imagine taking a much coarser grid or a larger time-step given the flow velocities and ingot size involved. Therefore, simulations such as the present one can only be performed on a research basis.

\section{RESULTS AND DISCUSSION}

The results of the simulation of the ingot cast and analyzed at Lukens Steel Company consist of time evolutions of the temperature field, the solid fraction field, the melt velocities, and the concentration fields for each element. The latter (carbon and sulfur only) are compared to the macrosegregation measurements.

Representative temperature fields at $t=3000$, 9000, 15,000, and 24,000 seconds are shown in Figures 2(a) through (d), respectively. These temperature fields are similar to those expected from a pure heat conduction analysis. Overall, the patterns indicate a favorable solidification behavior, and the isotherms are sufficiently inclined and rounded to avoid premature bridging at the centerline. Figure 3 shows the predicted cooling curve at the point of last solidification. This point is located in the hot top at the centerline, $0.221 \mathrm{~m}$ above the hot top junction. Superimposed on the cooling curve is the predicted solid fraction evolution at the same location. The cooling curve shows a sharp kink when the melt reaches the liquidus temperature of $1762 \mathrm{~K}$ corresponding to the initial composition (at $t \approx 3000 \mathrm{sec}$ onds). Beyond this time, the temperature drops only slightly due to the release of latent heat in the ingot, until solidification is complete and continued cooling causes the temperature to decrease more sharply. The cooling curve in Figure 3 can be used to estimate the total solidification time of the ingot. However, the exact time is difficult to determine, because a solidus temperature is not specified in the present

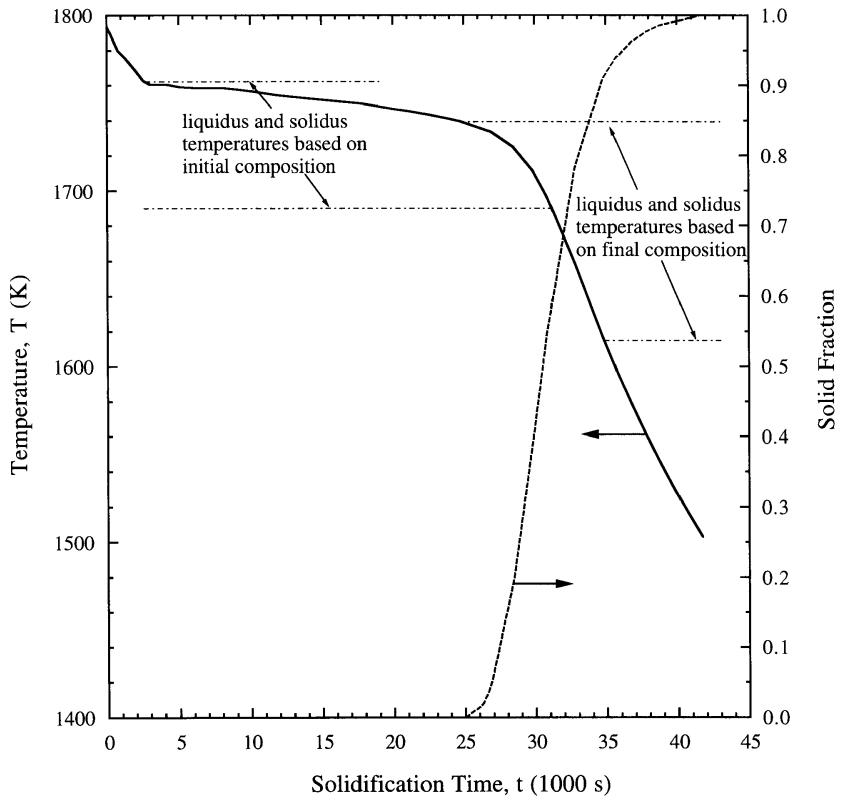

Fig. 3.-Predicted cooling curve and solid fraction evolution at the point of last solidification (at the centerline, $221 \mathrm{~mm}$ above the hot top junction); the liquidus and solidus temperatures indicated on the plot were calculated using the model of Miettinen. ${ }^{[22]}$

model. The microsegregation model, coupled with the macroscopic transport equations, predicts the solid fraction evolution, but the path predicted for the final 5 to 10 pct of liquid is likely to be unreliable due to the neglect of the formation of any secondary phases, such as eutectics, that can form at the end of solidification when the remaining liquid films are highly enriched in solutes. Therefore, we have used the microsegregation model for steel of Miettinen $^{[22]}$ to obtain an estimate of a realistic solidus temperature.

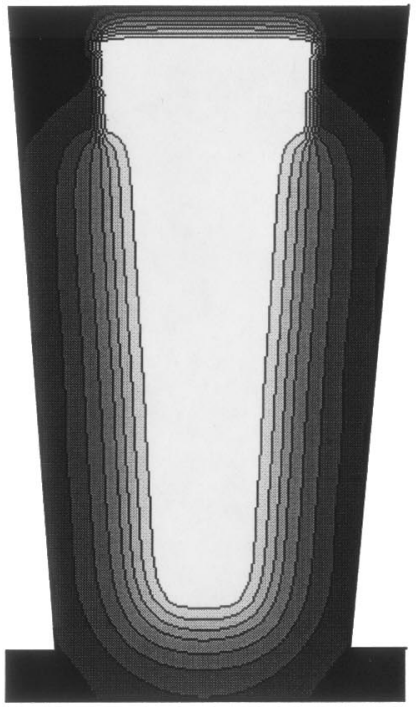

(a)

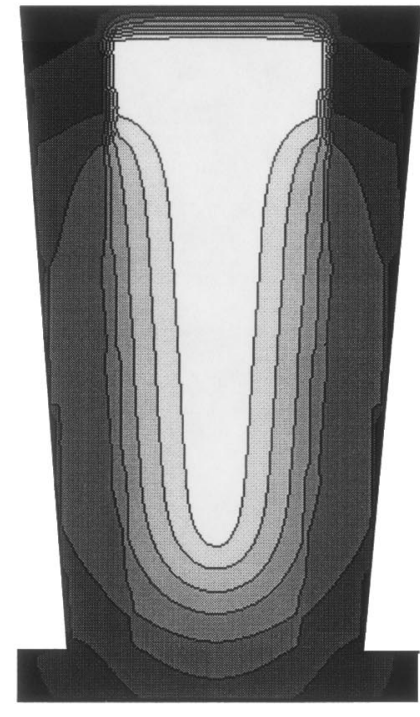

(b)

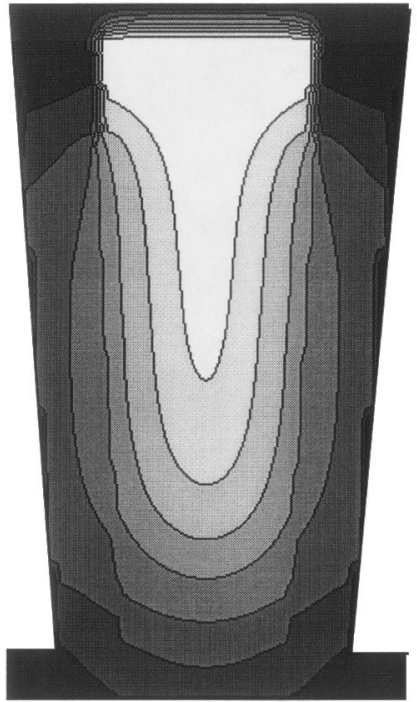

(c)

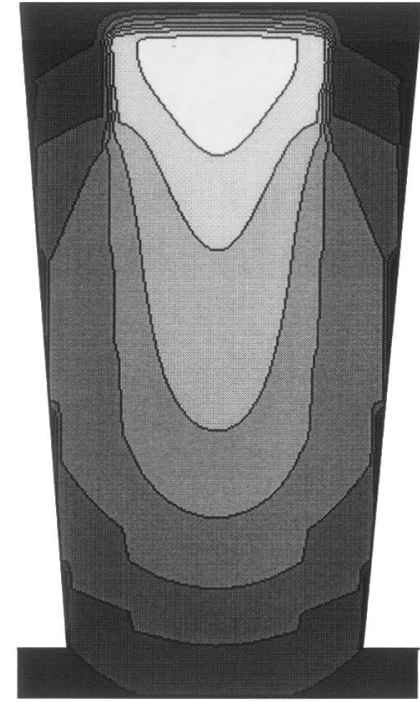

$(d)$

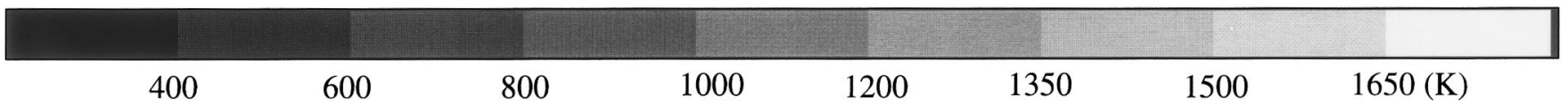

Fig. 2.-Predicted temperature fields at times of (a) $3000 \mathrm{~s}$, (b) $9000 \mathrm{~s},(c) 15,000 \mathrm{~s}$, and (d) 24,000 s. 
This model involves detailed thermodynamic and diffusion calculations for a representative closed volume element in the mushy zone, and was shown to provide solidus temperatures that are in close agreement with differential thermal analysis (DTA) measurements on a large variety of steels. Using the initial composition of the present steel, an estimated cooling rate of $0.02{ }^{\circ} \mathrm{C} / \mathrm{s}$, and a secondary dendrite arm spacing of $400 \mu \mathrm{m}$, the Miettinen model yields a solidus temperature of $1689 \mathrm{~K}$. With reference to Figure 3, this temperature then gives a total solidification time of 31,000 seconds (8.6 hours). That time agrees well with the "experimental" estimate by Lukens Steel Company of "less than" 9 hours. Note that beyond this time, the cooling curve in Figure 3 becomes linear, indicating that most solidification is indeed predicted to be completed. The preceding agreement in the total solidification time is similar to what other ingot heat-transfer models that explicitly specify a solidus temperature achieve (for example, Reference 9).

However, a closer examination of the predicted solid fraction evolution in Figure 3 reveals that solidification at the specified location in the ingot does not start until the temperature reaches about $1740 \mathrm{~K}(t=25,000$ seconds $)$, which is more than $20 \mathrm{~K}$ below the liquidus temperature corresponding to the initial steel composition, and does not reach values of about 0.9 until the temperature is about $1615 \mathrm{~K}(t=35,000$ $\mathrm{s}$ ), which is more than $70 \mathrm{~K}$ below the solidus temperature calculated by the Miettinen ${ }^{[22]}$ model using the initial steel composition (refer to previous discussion). These differences can be explained by macrosegregation. As is shown in the following figures, the point of last solidification in the ingot is highly segregated and the final composition at this location is predicted to be (refer to subsequent discussion) $0.70 \mathrm{pct}$ C, 0.96 pct $\mathrm{Mn}, 0.026$ pct $\mathrm{P}, 0.082$ pct $\mathrm{S}, 0.13$ pct $\mathrm{Cu}, 0.33$ pet Si, 0.19 pet Ni, 1.08 pct Cr, 0.24 pct Mo, 0.053 pct V, and 0.012 pct $\mathrm{Al}$ (compared to 0.41 pct $\mathrm{C}$, etc., Table I.). Using again the Miettinen model, but this time with the preceding final composition, yields a liquidus temperature of $1739 \mathrm{~K}$ and a solidus temperature of $1618 \mathrm{~K}$. Those two temperatures closely correspond with the start and end of solidification predicted by the present model, as shown in Figure 3 (disregarding the final few percent of solidification). Hence, macrosegregation is found to cause considerable shifts in the liquidus and solidus temperatures, and fixing the solidification range, as in all previous ingot heat-transfer models, can lead to considerable errors, especially in estimating the total solidification time (more than 1 hour in the present case). In general, solidification of the last few percent of liquid steel in a mushy zone is difficult to model accurately, and DTA measurements are not believed to always reflect the true solidus temperature. ${ }^{[23]}$ With the shifts possible due to macrosegregation, these uncertainties in the solidus temperature can add up to significant differences in the solidification time estimated by various methods and models.

Figures 4(a) through (d) show the predicted melt velocities and solid fraction contours at $t=500,3000,9000$, and 15,000 seconds, respectively. For better visualization, the velocity vectors were interpolated onto a coarser grid than used in the simulation. The solid fraction contours are plotted in increments of 0.2 . At $t=500$ seconds, solidification has just started along the mold walls and the mushy zone extends over less than 20 pct of the ingot. Due to primarily thermal buoyancy forces, there exist strong counterclockwise rotating convection cells. In the thermal boundary layer along the mush-liquid interface, the downward flowing melt reaches velocities of several centimeters per second. At subsequent times, as the mushy zone grows in size, the melt velocities decrease considerably, until at about $t=15,000$ seconds the single-phase liquid region disappears and all remaining flow is through the mush. In general, the velocities in the mush are several orders of magnitude smaller than in the pure liquid region, because of the friction the melt experiences as it flows through the dendritic network. Nonetheless, the flow in the mush is responsible for the development of macrosegregation, as shown subsequently. A more detailed discussion of the relative importance of thermal and solutal buoyancy forces in steel solidification can be found in Schneider and Beckermann. ${ }^{[18]}$

Also apparent from Figure 4 is the development of the shrinkage cavity at the top of the ingot. At $t=15,000$ seconds, the shrinkage cavity is close to its final shape. This shape and the predicted depth at the centerline (measured form the original fill height) of about $0.14 \mathrm{~m}$ agree closely with the cavity dimensions observed for the ingot cast at Lukens Steel Company.

The evolution of macrosegregation is illustrated in Figures 5(a) through (d) at the same four times as the velocities in Figure 4. Plotted is the predicted mixture (solid and liquid) concentration of carbon normalized by the nominal carbon concentration $(0.41 \mathrm{pct})$ with the maximum and minimum values provided below each plot. It is shown in Schneider and Beckermann ${ }^{[18]}$ that the macrosegregation patterns for all elements look similar if scaled by the initial concentration and the partition coefficient. Therefore, only the patterns for carbon are presented here. It can be seen that the normalized concentration field evolves from initially uniform values of unity to a nonuniform pattern where the solute is highly redistributed. Since the solute redistribution is due to melt flow in the mushy zone, the pattern development occurs simultaneously with the growth of the mushy zone (Figure 4). The flow advects solute-rich melt out of the mushy zone, leading to a continual enrichment of the remaining melt in the single-phase liquid region. Consequently, the outer regions and the lower part of the ingot are generally negatively segregated, while the last part to solidify, i.e., the region in and near the centerline of the hot top, has the highest positive macrosegregation.

The resulting final macrosegregation patterns in the fully solidified ingot are shown in Figures 6(a) and (b) for carbon and sulfur, respectively. Note that a gray scale different from Figure 5 is used in order to better visualize the pattern in the top region. The patterns for the two elements indeed look similar, although local differences are possible. On the average, the normalized macrosegregation values for sulfur are about 1.8 times the carbon values. The same factor was found by Schneider and Beckermann ${ }^{[18]}$ for a different steel composition, who showed that the root-mean-square of macrosegregation for each element can be linearly related to the partition coefficients. As opposed to the simulations of Schneider and Beckermann, which dealt with a hypothetical steel ingot only $20 \times 10 \mathrm{~cm}$ in size, no A segregates are predicted in the present simulation, although the sulfur prints revealed their presence in the ingot cast (Section II). This can be directly attributed to the lack of a sufficiently fine 


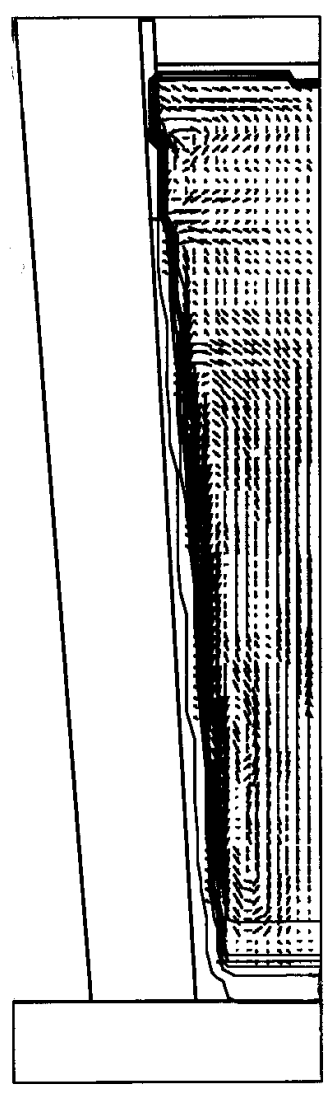

$=2.500 \mathrm{e}-02$

(a)

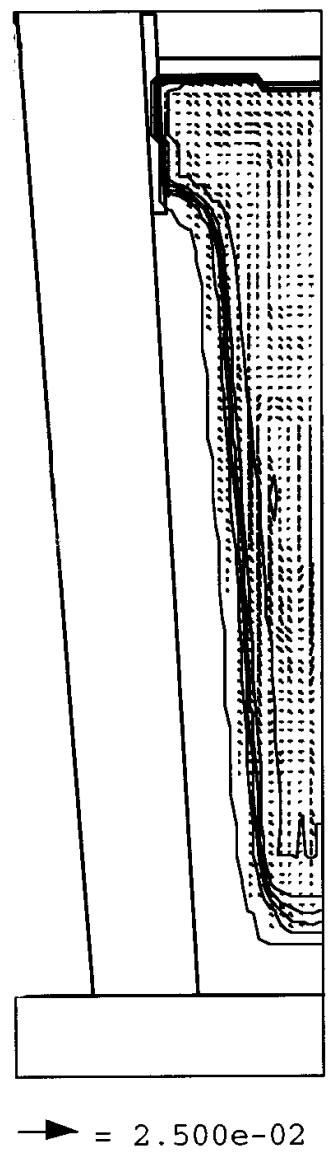

(b)

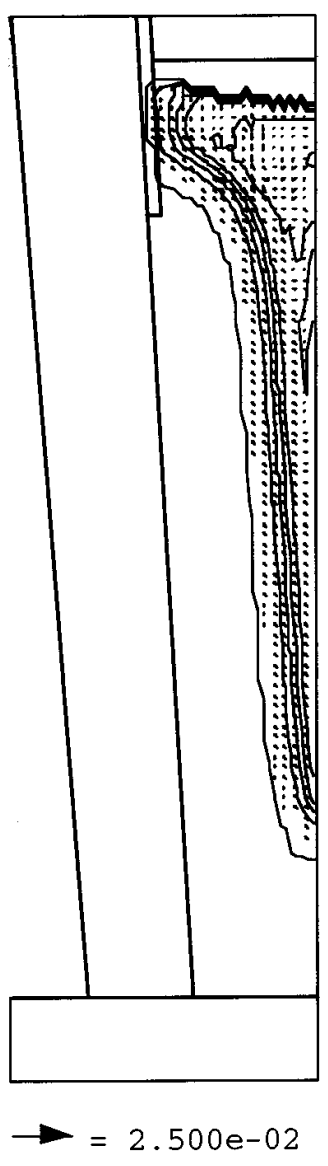

(c)

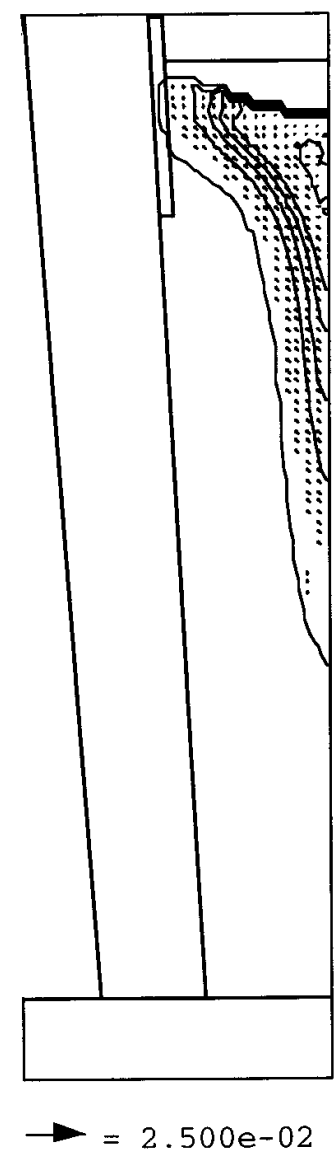

(d)

Fig. 4.-Predicted velocity vectors (in m/s) and solid fraction contours (in 20 pct increments) at times of (a) $500 \mathrm{~s},(b) 3000 \mathrm{~s},(c) 9000 \mathrm{~s}$, and (d) $15,000 \mathrm{~s}$.

numerical grid. Similar observations were made by Vannier et al. ${ }^{[17]} \mathrm{A}$ control volume used in the computations is about $18 \times 55-\mathrm{mm}$ large, while an A segregate is typically only several primary dendrite arm spacings in diameter (i.e., of the order $5 \mathrm{~mm}$ ). In order to predict A segregates in the large ingot of the present study, we estimate that the grid spacing would need to be decreased by at least a factor of 30 in each direction. The resulting increase in computational cost by more than two orders of magnitude would make such refined simulations impossible for the near future. As mentioned in Section I, A segregates can have an effect on macrosegregation that extends beyond the region where they are visible in the solidified ingot. Only a comparison with experimental data, as is presented next, can help to clarify the importance of this effect.

Figures 7(a) and (b) show a comparison of the measured and predicted carbon and sulfur, respectively, macrosegregation levels along the vertical centerline of the ingot where the bulk of the measurements were taken. In general, the agreement between the measured and predicted concentrations can be considered good, although some obvious differences are present. The good agreement is particularly noteworthy in view of the fact that there are essentially no adjustable parameters in the model (the largest uncertainty perhaps being the thermal boundary conditions). Even the mushy zone permeability relation (Eq. [1]). which is known to have a large effect on macrosegregation predictions, ${ }^{[12]}$ cannot be considered to be adjustable, because the same relation has been found to work well for other alloy systems (Figure 4 in Reference 21 for a comparison of the relation with experimental data), and the correlation used to calculate the primary dendrite arm spacing in the permeability relation is well established. Particularly good is the agreement between the measured and predicted sulfur concentrations inside the hot top (Figure 7(b)). The agreement is not quite as good for carbon (Figure 7(a)), for which the measured concentrations at the top are consistently above the predicted values. This discrepancy is somewhat surprising, because the model is formulated such that the concentration predictions for one element must be as good (or bad) as for any other solute. While the predicted carbon and sulfur concentrations along the entire centerline are different by a factor of 1.8 (as discussed previously), the measured values at the very top are not. In lieu of any measurements for a third element, we believe that the higher measured carbon concentrations near the very top may be due to pick up of carbon from the hot topping flux combination. It should also be noted that the proper modeling of the shrinkage cavity at the top appears from Figure 7 to be crucial in predicting the macrosegregation pattern in the hot top region. Without the shrinkage cavity, the predicted pattern in the upper part of the ingot would be shifted upward by almost half of the original hot top height $(140 \mathrm{~mm})$, and the agreement with the measurements would be unacceptable. The slight decrease in the predicted concentrations just below the top surface is due to the fact that the point of last solidification is several 


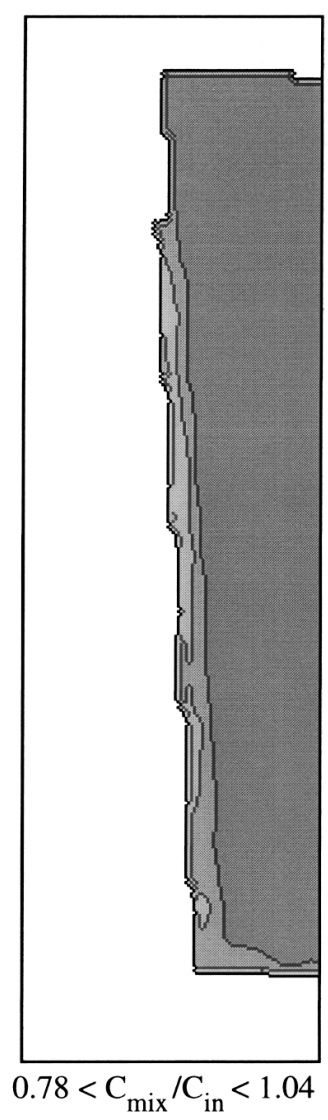

(a)

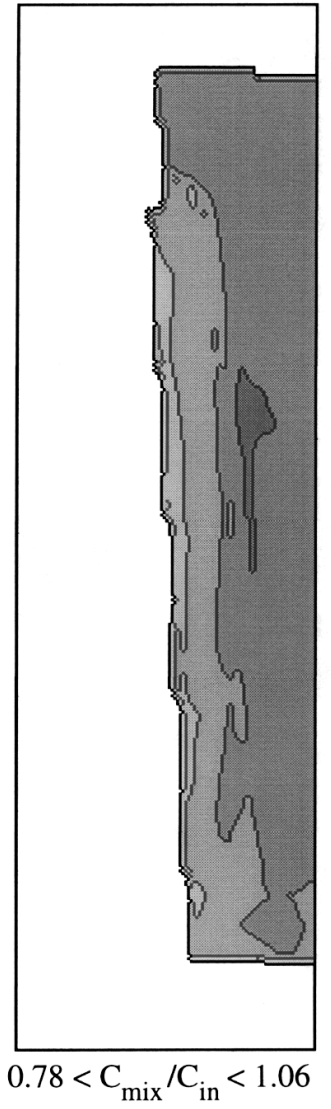

(b)

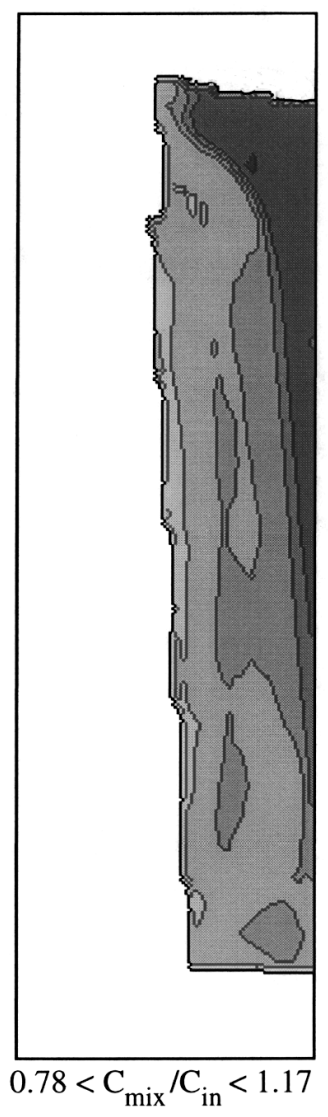

(c)

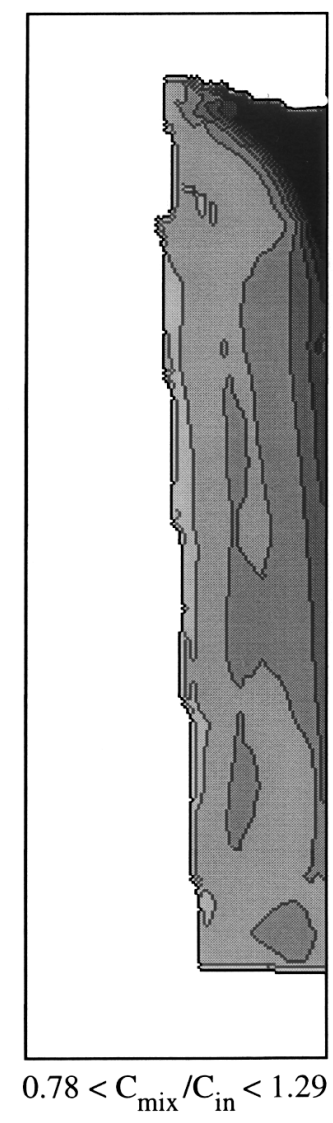

(d)

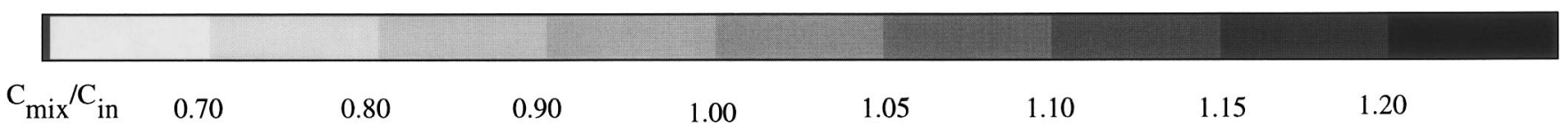

Fig. 5-Predicted mixture concentrations of carbon, $C_{\text {mix }}$, normalized by the initial (ladle) concentration, $C_{i n}$, at times of $(a) 500 \mathrm{~s}$, $(b) 3000 \mathrm{~s}$, $(c) 9000 \mathrm{~s}$, and $(d) 15000 \mathrm{~s}$.

centimeters below the surface. This effect is not reflected by the measurements.

The measured concentrations reach the nominal values about $0.8 \mathrm{~m}$ below the hot top junction, indicate negative macrosegregation of about 10 pct for carbon and 18 pct for sulfur over the lower part of the ingot, and appear to approach the nominal values again at the very bottom (Figure 7). The predicted macrosegregation, on the other hand, remains positive over the entire ingot centerline, except for the bottom $0.25 \mathrm{~m}$, where it becomes slightly negative. The predicted negative region at the very bottom is due to the flow of solute-rich liquid out of the mush that forms at the walls in the early stages of solidification, and such negative segregation is also observed adjacent to the vertical mold walls (Figure 6). The disagreement between the measured and predicted centerline concentrations over the lower two-thirds of the ingot is most likely due to the neglect of sedimentation of free equiaxed crystals in the model. The free crystals form early in the solidification process and are poor in solutes. When they settle, a zone of negative macrosegregation develops in the lower center part of the ingot. In fact, the sulfur prints of the ingot cast revealed that the measured zone of negative macrosegregation coincides with an equiaxed grain structure, while the remainder of the ingot is columnar. When the equiaxed crystals settle, a corresponding volume of solute-rich melt must flow upward, further contributing to the positive macrosegregation in the top portion of the ingot. This upflow of melt due to settling of solid may also explain why the measured carbon concentrations in the top one-third of the ingot are consistently larger than the values predicted by the present model (which assumes a stationary solid) (Figure 7(a)). For sulfur (Figure 7(b)), the measured concentrations around the hot top junction are also larger than the predicted ones, although the effect is not as obvious due to larger fluctuations in the measurements. As discussed previously, the agreement between the measured and predicted sulfur concentrations becomes good again well inside the hot top. In summary, sedimentation of equiaxed crystals, and the corresponding upflow of melt, appears to be the most logical explanation for the discrepancies between the measured and predicted centerline macrosegregation. This explanation is consistent with the findings of Olsson et al. ${ }^{[5]}$ who also present a simple model for calculating the extent of macrosegregation due to settling of crystals. It is also shown by Olsson et al. that melt convection, as modeled in the present study, and convection through A-segregate channels affect primarily the macrosegregation level in the top portion of the ingot. Since the agreement between the 


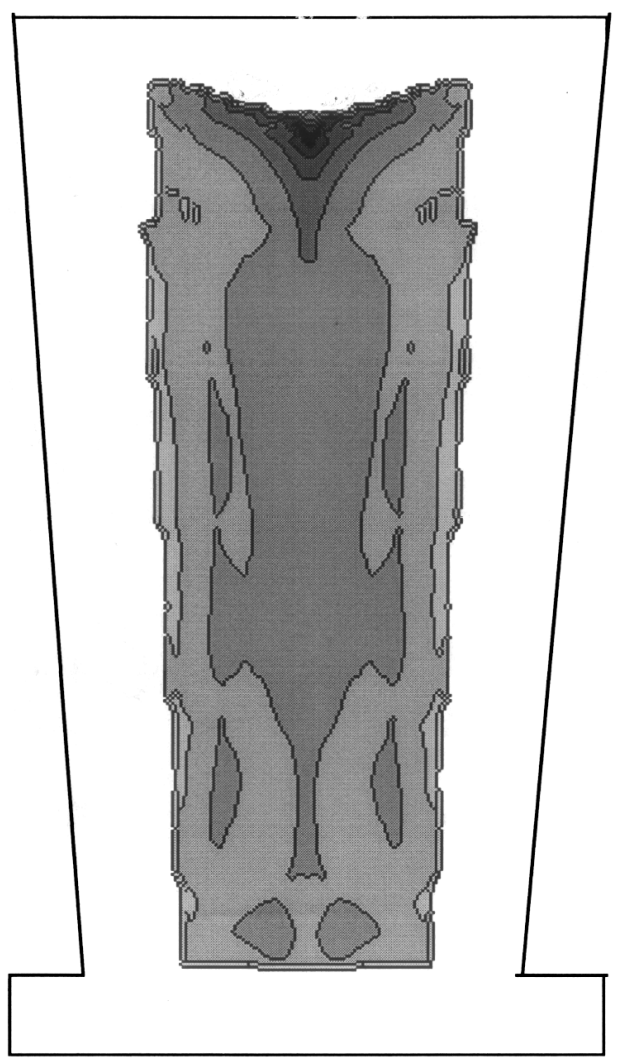

$0.78<\mathrm{C}_{\text {mix }} / \mathrm{C}_{\text {in }}<1.71$

(a)

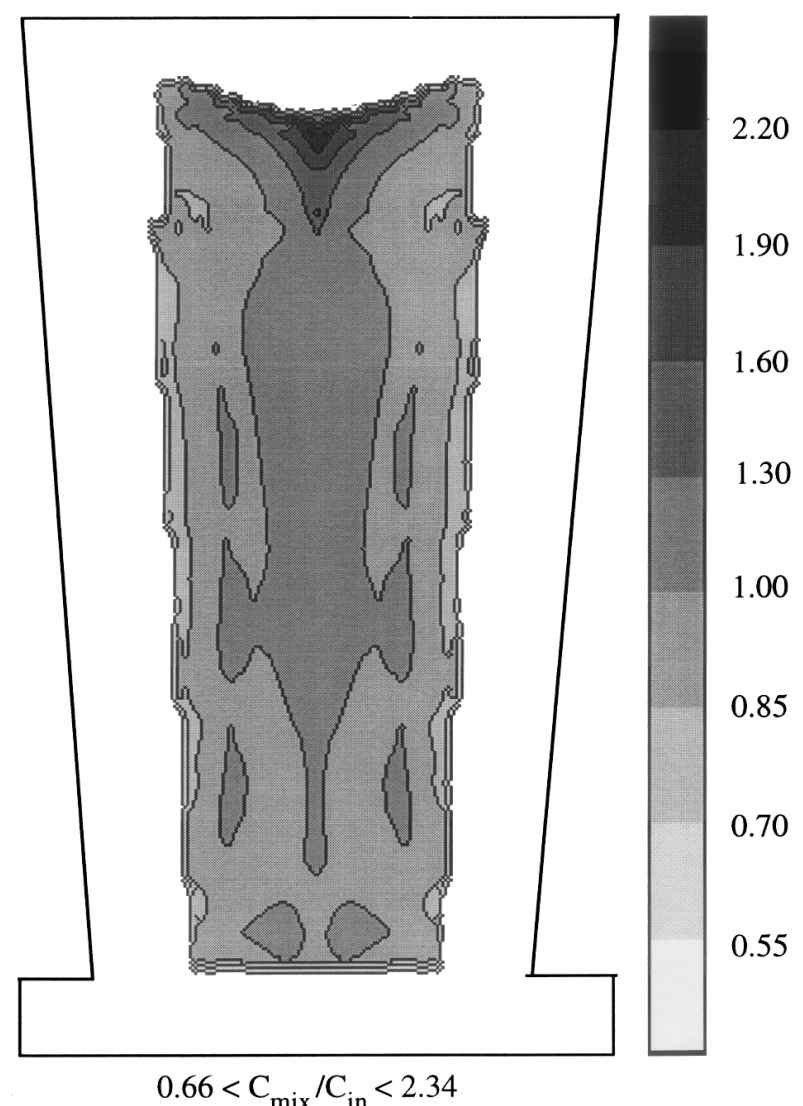

(b)

Fig. 6.-Predicted final macrosegregation pattern for $(a)$ carbon and $(b)$ sulfur.

measurements and predictions near the top is good (at least for sulfur), it is unlikely that the lack of A segregates in the present simulation contributes to any of the discrepancies in the lower part of the ingot.

\section{CONCLUSIONS}

A numerical simulation is reported of the heat transfer, species transport, and melt convection during solidification of a large steel ingot. The study represents a first attempt at applying a fully coupled, multicomponent solidification with melt convection model to an industrial-scale casting. It is also one of the very few studies that compares measured macrosegregation patterns to predictions from a model that has essentially no adjustable parameters. Major shortcomings of the simulation are (1) the neglect of sedimentation of free equiaxed crystals, (2) the coarseness of the numerical grid preventing the direct prediction of A segregates, and (3) the excessively long computational time.

The simulation results reveal the nature of the heat transfer, melt flow, and species transport phenomena during solidification of the ingot. The comparison between the measurements and predictions allows for the following main conclusions.

1. The predicted level and variation of the macrosegregation along the vertical centerline are generally in good agreement with the measurements.

2. The neglect of the sedimentation of free equiaxed crystals prevents the prediction of the measured negative macrosegregation in the bottom half of the ingot; it also leads to a corresponding underprediction of the positive macrosegregation near the hot top junction.

3. The absence of $\mathrm{A}$ segregates in the present simulation (due to the coarse grid) apparently does not cause major disagreements along the centerline.

4. The pickup of carbon from the insulation material leads to an underprediction of the measured positive carbon macrosegregation at the very top of the ingot (in the hot top); no such disagreement exists for sulfur.

5. Macrosegregation leads to considerable shifts (more than $50 \mathrm{~K}$ ) in the temperatures at which solidification is complete; consequently, the predicted final solidification time is much different from what is obtained from simple heat transfer or even uncoupled macrosegregation models that assume a fixed solidus temperature.

6. The predicted shape and depth of the shrinkage cavity at the top agree well with observations made on the cast ingot; the modeling of the shrinkage cavity is crucial in predicting the correct macrosegregation variation in and near the hot top.

As reviewed in Beckermann and Wang, ${ }^{[12]}$ models that include the settling of equiaxed grains have recently become available, but are preliminary in some aspects. In particular, the theoretical description of melt undercooling and the initial formation of free grains in the presence of melt convection need additional research attention before such model can 


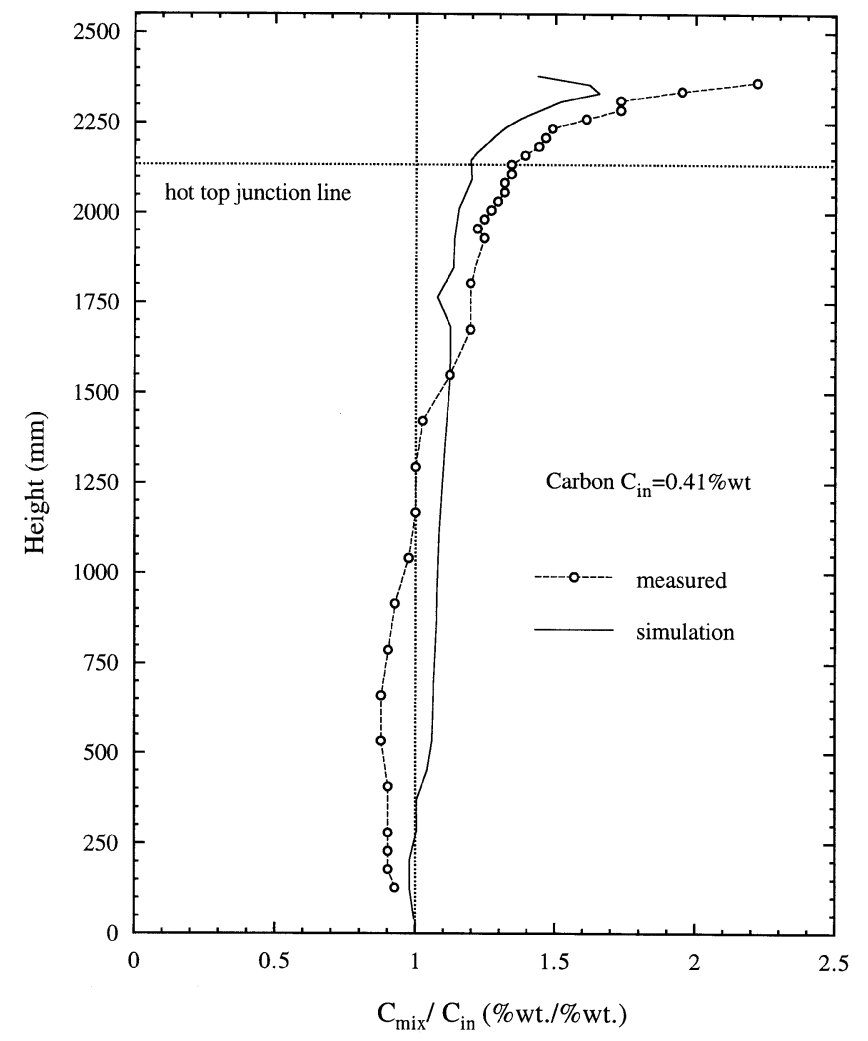

(a)

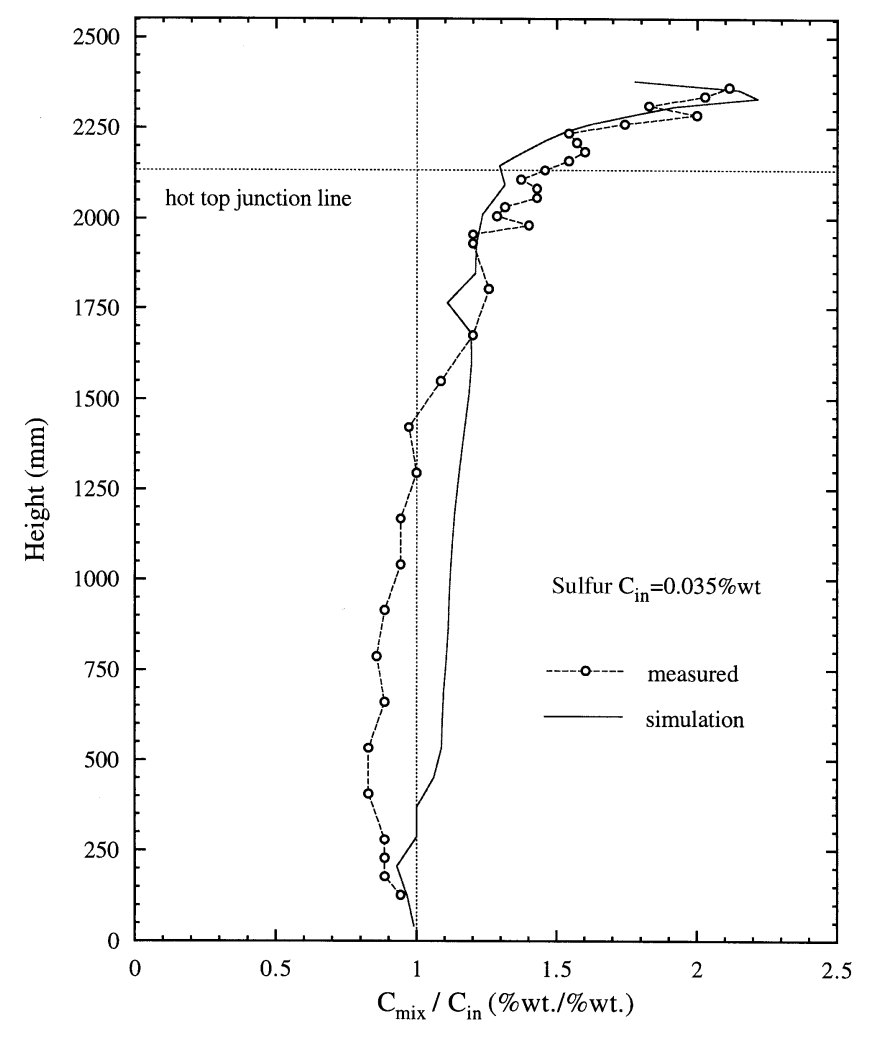

(b)

Fig. 7.-Comparison of measured and predicted macrosegregation variation of $(a)$ carbon and $(b)$ sulfur along the vertical centerline of the ingot.

be applied to ingot casting. It is hoped that the measurements presented here can serve as a test case for validating such future models.

\section{ACKNOWLEDGMENTS}

The support of the Lukens Steel Company, in particular, Mr. H.E. Woodruff, is gratefully acknowledged. Partial support was also provided by the National Science Foundation under Grant No. CTS-9501389.

\section{REFERENCES}

1. M.C. Flemings: Solidification Processing, McGraw-Hill, New York, NY, 1974.

2. A. Hultgren: Scand. J. Met., 1973, vol. 2, p. 217

3. M.C. Flemings: Scand. J. Met., 1976, vol. 5, pp. 1-15.

4. H. Fredriksson: Cand. Metall. Q., 1991, vol., 30, pp. 235-44.

5. Olsson, R. West, and H. Fredriksson: Scand. J. Met., 1986, vol. 15, pp. 104-12.

6. R. Mehrabian and M.C. Flemings: Metall. Trans., 1970, vol. 1, pp. $455-64$.

7. T. Fujii, D.R. Poirier, and M.C. Flemings: Metall. Trans. B, 1979, vol. 10B, pp. 331-39.

8. I. Ohnaka: in "State of the Art of Computer Simulation of Casting and Solidification Processes," H. Fredriksson, ed., E-MRS, Materials Research Society, Pittsburgh, PA, 1986, pp. 211-23.

9. B.G. Thomas, I.V. Samarasekera, and J.K. Brimacombe: Metall. Trans. A, 1987, vol. 18B, pp. 119-30.
10. W.D. Bennon and F.P. Incropera: Metall. Trans. B, 1987, vol. 18B, pp. 611-16.

11. C. Beckermann and R. Viskanta: Physicochem. Hydrodyn., 1988, vol. 10, pp. 195-213.

12. C. Beckermann and C.Y. Wang: in Annual Review of Heat Transfer, C.L. Tien, ed., Begell House Inc., New York, NY, 1995, vol. 6.

13. P.J. Prescott and F.P. Incropera: in Advances in Heat Transfer, J.P. Hartnett, T. Irvine, Y.I. Cho, and G.A. Green, eds., Academic Press, New York, NY, 1996, vol. 28, pp.231-38.

14. F. Roch, H. Combeau, I. Poitrault, J.C. Chevrier, and G. Lesoult: Proc. 6th Int. Iron and Steel Congr., vol. 1, Fundamentals, Iron and Steel Institute of Japan, Nagoya, 1990, pp. 665-72.

15. H. Combeau, F. Roch, I. Poitrault, J.C. Chevrier, and G. Lesoult: in Advanced Computational Methods in Heat Transfer, vol. 3, Phase Change and Combustion Simulation, Springer-Verlag, New York, NY, 1990, pp. 79-90.

16. F. Roch, H. Combeau, J.C. Chevrier, and G. Lesoult: in Modeling of Casting, Welding and Advanced Solidification Processes V, TMS, Warrendale, PA, 1991, pp. 789-95.

17. H. Vannier, H. Combeau, and G. Lesoult: in Numerical Methods in Industrial Forming Processes, A.A. Balkema, ed., Rotterdam, The Netherlands, 1992, pp. 835-40.

18. M.C. Schneider and C. Beckermann: Metall. Mater. Trans. A, 1995, vol. 26A, pp. 2373-88.

19. A. Kagawa and T. Okamoto: Mater. Sci. Technol., 1986, vol. 2, pp. 997-1008.

20. H. Jacobi and K. Schwerdtfeger: Metall. Trans. A, 1976, vol. 7A, pp. 811-20.

21. M.C. Schneider, J.P. Gu, C. Beckermann, W.J. Boettinger, and U.R Kattner: Metall. Mater. Trans. A, 1997, vol. 28A, pp. 1517-31.

22. J. Miettinen: Metall. Trans. A, 1992, vol. 23A, pp. 1155-70.

23. A.A. Howe: Ironmaking and Steelmaking, 1988, vol. 15, pp. 134-42. 